International Journal of Pure and Applied Mathematics

Volume 93 No. 2 2014, 233-242

ISSN: 1311-8080 (printed version); ISSN: 1314-3395 (on-line version)

url: http://www.ijpam.eu

doi: http://dx.doi.org/10.12732/ijpam.v93i2.8

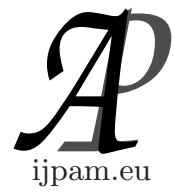

\title{
SOFT EXPERT GENERALIZED CLOSED SETS
}

\author{
K. Kannan ${ }^{1}$, D. Narasimhan ${ }^{2}$ \\ ${ }^{1,2}$ Department of Mathematics \\ Srinivasa Ramanujan Centre \\ SASTRA University \\ Kumbakonam, Tamil Nadu, 612 001, INDIA
}

\begin{abstract}
The soft expert models are richer than soft set models since the soft set models are created with the help of one expert whereas but the soft expert models are made with the opinions of all experts. In the present paper, we introduce soft expert generalized closed sets (soft expert $g$-closed sets) and soft expert generalized open sets (soft expert $g$-open sets) in soft expert topological spaces and study their basic properties. A sufficient condition for a soft expert $g$-closed set to be a soft expert closed is also presented.
\end{abstract}

AMS Subject Classification: 06D72

Key Words: soft expert $g$-closed sets, soft expert $g$-open sets, soft expert open sets, soft expert closed sets

\section{Introduction}

Several set theories such as theory of vague sets, theory of interval mathematics $[2,5]$, theory of fuzzy sets [18], theory of intuitionistic fuzzy sets [1] and theory of rough sets [15] can be used as tools for dealing with uncertainties, but all these theories have their own difficulties. According to Molodtsov in [13], inadequacy of the parametrization tool of the theory is the main reason for these difficulties. To overcome this, he initiated the concept of soft set theory as a new mathematical tool which is free from the problems mentioned above and presented the fundamental results of the new theory and successfully applied it

Received: February 13, 2014

(C) 2014 Academic Publications, Ltd.

$\S$ Correspondence author url: www.acadpubl.eu 
to several directions such as smoothness of functions, game theory, operations research, Riemann-integration, Perron integration, theory of probability etc.

A soft set is a collection of approximate descriptions of an object. Recent research works on soft set theory and its applications in various fields are progressing rapidly. An application of soft sets in decision making problems was presented by Maji et al $[10,11]$ based on the reduction of parameters to keep the optimal choice objects. Chen [3] introduced a new approach of soft set parametrization reduction and a comparison of it with attribute reduction in rough set theory. Soft sets are a class of special information systems which was shown by Pei and Miao [16].

Soft topological spaces over an initial universe with a fixed set of parameters was introduced by Muhammad Shabir and Munazza Naz [14] and then many new notions, like, soft open sets, soft closed sets, soft closure, soft interior points, soft neighborhood of a point and soft separation axioms were investigated by them. Levine $[8,4]$ introduced generalized closed sets and $T_{\frac{1}{2}}$-spaces in topological spaces. Kannan [7] introduced soft generalized closed sets in the context of soft topological spaces and studied the new separation axiom, called soft $T_{\frac{1}{2}}$-spaces.

Mahanta and Das [12] introduced the concepts of soft semi open sets and soft semi closed sets in soft topological spaces whereas Levine [9] introduced semi open and semi closed sets in the context of topological spaces.

Shawkat Alkhazaleh and Abdul Razak Salleh [17] defined soft expert sets and created the model in which user can know the opinion of all experts in one model. Sabir Hussain [6] introduced soft expert topological spaces which are defined over an initial universe with a fixed set of parameters and with the opinion of all expert instead of only one expert. The notions of soft expert open sets, soft expert closed sets, soft expert closure, soft expert interior, soft expert exterior and soft expert boundary are introduced and their basic properties are investigated. Finally, these notions have been applied to the problem of decision-making.

The present paper is mainly concerned with soft expert generalized closed sets and their basic properties in soft expert topological spaces which is introduced with the help of soft expert open sets.

\section{Preliminaries}

Let $U$ be an initial universe, $E$ be a set of parameters and $X$ be the set of opinions, $Z=E \times X \times O$ and $A \subseteq Z$. Let $P(U)$ denote the power set of $U$ and 
$A$ be a non-empty subset of $Z$. A pair $(F, A)$ is called a soft expert set over $U$, where $F$ is a mapping given by $F: A \rightarrow P(U)$.

Clearly, a soft expert set is not a set. For two soft sets $(F, A)$ and $(G, B)$ over a common universe $U$, we say that $(F, A)$ is a soft subset of $(G, B)$ if (1) $A \subseteq B$ and (2) for all $e \in B, G(e) \subseteq F(e)$. We write $(F, A) \widetilde{\subseteq}(G, B)$. $(F, A)$ is said to be a soft superset of $(G, B)$, if $(G, B)$ is a soft subset of $(F, A)$. We denote it by $(F, A) \supseteq(G, B)$. Two soft sets $(F, A)$ and $(G, B)$ over a common universe $U$ are said to be soft equal if $(F, A)$ is a soft subset of $(G, B)$ and $(G, B)$ is a soft subset of $(F, A)$.

The NOT set of $Z=E \times X \times O$ denoted by $Z$, is defined by $Z=\left\{\left(e_{i}, x_{j}, o_{k}\right)\right.$, for all $i, j, k\}$ where $e_{i}$ is not $e_{i}$. The complement of a soft expert set $(F, A)$ is denoted by $(F, A)^{\prime}$ and is defined by $(F, A)^{\prime}=\left(F^{\prime}, A\right)$ where $F^{\prime}: A \rightarrow P(X)$ is a mapping given by $F^{\prime}(e)=X-F(e)$ for all $e \in A$.

The union of two soft sets of $(F, A)$ and $(G, B)$ over the common universe $U$ is the soft set $(H, C)$, where $C=A \cup B$ and for all $e \in C, H(e)=F(e)$ if $e \in A-B, G(e)$ if $e \in B-A$ and $F(e) \cup G(e)$ if $e \in A \cap B$. We write $(F, A) \cup(G, B)=(H, C)$. The following is the definition of the intersection of two soft sets. The intersection $(H, C)$ of two soft sets $(F, A)$ and $(G, B)$ over a common universe $U$, denoted $(F, A) \cap(G, B)$, is defined as $C=A \cap B$, and $H(e)=F(e) \cap G(e)$ for all $e \in C$.

Let $x \in U$, then $(x, A)$ denotes the soft expert set over $U$ for which $x(e)=$ $\{x\}$, for all $e \in A$. Let $(F, A)$ be a soft expert set over $U$ and $V$ be a non-empty subset of $U$. Then the sub soft expert set of $(F, A)$ over $V$ denoted by $\left(V_{F}, A\right)$, is defined as follows: $V_{F}(e)=V \cap F(e)$, for all $e \in A$. In other words $\left(V_{F}, E\right)$ $=\widetilde{V} \cap(F, A)$.

Let $\tau$ be the collection of soft expert sets over $U$, then $\tau$ is said to be a soft expert topology on $U$ if (1) $\phi, \widetilde{U}$ belong to $\tau,(2)$ the union of any number of soft expert sets in $\tau$ belongs to $\tau,(3)$ the intersection of any two soft expert sets in $\tau$ belongs to $\tau$. The triplet $(U, \tau, A)$ is called a soft expert topological space over $U$. Let $(U, \tau, A)$ be a soft expert space over $U$, then the members of $\tau$ are said to be soft expert open sets in $U$.

Let $(U, \tau, A)$ be a soft expert space over $U$. A soft expert set $(F, A)$ over $U$ is said to be a soft expert closed set in $U$, if its complement $(F, A)^{\prime}$ belongs to $\tau$. Let $\tau=\{\phi, \widetilde{U}\}$. Then $\tau$ is called the soft expert indiscrete topology on $U$ and $(U, \tau, A)$ is said to be a soft indiscrete space over $U$. Let $\tau$ be the collection of all soft expert sets which can be defined over $U$. Then $\tau$ is called the soft expert discrete topology on $U$ and $(U, \tau, A)$ is said to be a soft expert discrete space over $U$.

Let $(U, \tau, A)$ be a soft expert topological space over $U$ and $(F, A)$ be a soft 
expert set over $U$. Then, the soft expert closure of $(F, A)$, denoted by $\overline{(F, A)}$ is the intersection of all soft expert closed supersets of $(F, A)$. Clearly $\overline{(F, A)}$ is the smallest soft expert closed set over $U$ which contains $(F, A)$. The soft expert interior of $(F, A)$, denoted by $(F, A)^{0}$ is the union of all soft expert open subsets of $(F, A)$. Clearly $(F, A)^{0}$ is the largest soft expert open set over $U$ which is contained in $(F, A)$.

\section{Soft Expert Generalized Closed Sets}

In this section, we define and study the concept of soft expert generalized closed sets in soft expert topological spaces. Now, we begin this section by introducing soft expert generalized closed sets (soft expert $g$-closed sets) in soft expert topological spaces.

Definition 3.1. A soft set $(F, A)$ in a soft expert topological space $(U, \tau, A)$ over $U$ is called soft expert generalized closed (soft expert $g$-closed) if $\overline{(F, A)} \widetilde{\subseteq}(G, A)$ whenever $(F, A) \widetilde{\subseteq}(G, A)$ and $(G, A)$ is soft expert open in $U$.

Example 3.2. Let $X=\{\mathrm{p}, \mathrm{q}, \mathrm{r}\}, U=\left\{u_{1}, u_{2}, u_{3}, u_{4}\right\}, E=\left\{e_{1}, e_{2}, e_{3}\right\}$. Suppose that $A=\left\{\left(e_{1}, p, 1\right),\left(e_{1}, q, 1\right),\left(e_{1}, r, 1\right),\left(e_{2}, p, 1\right),\left(e_{2}, q, 1\right),\left(e_{2}, r, 1\right)\right.$, $\left(e_{3}, p, 1\right),\left(e_{3}, q, 1\right),\left(e_{3}, r, 1\right),\left(e_{1}, p, 0\right),\left(e_{1}, q, 0\right),\left(e_{1}, r, 0\right),\left(e_{2}, p, 0\right),\left(e_{2}, q, 0\right)$, $\left.\left(e_{2}, r, 0\right),\left(e_{3}, p, 0\right),\left(e_{3}, q, 0\right),\left(e_{3}, r, 0\right)\right\}$.

Let $\tau=\{\widetilde{\phi}, \widetilde{U},(F, A),(G, A)\}$ where $(F, A)=\left\{\left(\left(e_{1}, p, 1\right),\left\{u_{1}, u_{2}, u_{4}\right\}\right)\right.$, $\left(\left(e_{1}, q, 1\right),\left\{u_{1}, u_{4}\right\}\right),\left(\left(e_{1}, r, 1\right),\left\{u_{3}, u_{4}\right\}\right),\left(\left(e_{2}, p, 1\right),\left\{u_{4}\right\}\right),\left(\left(e_{2}, q, 1\right),\left\{u_{1}, u_{3}\right\}\right)$, $\left(\left(e_{2}, r, 1\right),\left\{u_{1}, u_{2}, u_{4}\right\}\right),\left(\left(e_{3}, p, 1\right),\left\{u_{3}, u_{4}\right\}\right),\left(\left(e_{3}, q, 1\right),\left\{u_{1}, u_{2}\right\}\right),\left(\left(e_{3}, r, 1\right),\left\{u_{4}\right\}\right)$, $\left(\left(e_{1}, p, 0\right),\left\{u_{3}\right\}\right),\left(\left(e_{1}, q, 0\right),\left\{u_{2}, u_{3}\right\}\right),\left(\left(e_{1}, r, 0\right),\left\{u_{1}, u_{2}\right\}\right),\left(\left(e_{2}, p, 0\right),\left\{u_{1}, u_{2}, u_{3}\right\}\right)$, $\left(\left(e_{2}, q, 0\right),\left\{u_{2}, u_{4}\right\}\right),\left(\left(e_{2}, r, 0\right),\left\{u_{3}\right\}\right),\left(\left(e_{3}, p, 0\right),\left\{u_{1}, u_{2}\right\}\right),\left(\left(e_{3}, q, 0\right),\left\{u_{3}, u_{4}\right\}\right)$, $\left.\left(\left(e_{3}, r, 0\right),\left\{u_{1}, u_{2}, u_{3}\right\}\right)\right\}$ and $(G, A)=\left\{\left(\left(e_{1}, p, 1\right), u_{1}, u_{4}\right), \quad\left(\left(e_{1}, q, 1\right),\left\{u_{4}\right\}\right)\right.$, $\left.\left(\left(e_{2}, q, 1\right),\left\{u_{1}, u_{3}\right\}\right),\left(\left(e_{2}, r, 1\right),\left\{u_{1}\right\}\right),\left(\left(e_{1}, r, 0\right),\left\{u_{2}\right\}\right),\left(\left(e_{2}, p, 0\right),\left\{u_{1}, u_{3}\right\}\right)\right\}$.

Then $(U, \tau, A)$ is a soft expert topological space and $(H, A)=\left\{\left(\left(e_{1}, p, 1\right)\right.\right.$, $\left.\left\{u_{3}\right\}\right),\left(\left(e_{1}, q, 1\right),\left\{u_{2}, u_{3}\right\}\right),\left(\left(e_{1}, r, 1\right),\left\{u_{1}, u_{2}\right\}\right),\left(\left(e_{2}, p, 1\right),\left\{u_{1}, u_{2}, u_{3}\right\}\right),\left(\left(e_{2}, q, 1\right)\right.$, $\left.\left\{u_{2}, u_{4}\right\}\right),\left(\left(e_{2}, r, 1\right),\left\{u_{3}\right\}\right),\left(\left(e_{3}, p, 1\right),\left\{u_{1}, u_{2}\right\}\right),\left(\left(e_{3}, q, 1\right),\left\{u_{3}, u_{4}\right\}\right),\left(\left(e_{3}, r, 1\right)\right.$, $\left.\left\{u_{1}, u_{2}, u_{3}\right\}\right),\left(\left(e_{1}, p, 0\right),\left\{u_{1}, u_{2}, u_{4}\right\}\right),\left(\left(e_{1}, q, 0\right),\left\{u_{1}, u_{4}\right\}\right),\left(\left(e_{1}, r, 0\right),\left\{u_{3}, u_{4}\right\}\right)$, $\left(\left(e_{2}, p, 0\right),\left\{u_{4}\right\}\right),\left(\left(e_{2}, q, 0\right),\left\{u_{1}, u_{3}\right\}\right),\left(\left(e_{2}, r, 0\right),\left\{u_{1}, u_{2}, u_{4}\right\}\right),\left(\left(e_{3}, p, 0\right),\left\{u_{3}, u_{4}\right\}\right)$, $\left.\left(\left(e_{3}, q, 0\right),\left\{u_{1}, u_{2}\right\}\right),\left(\left(e_{3}, r, 0\right),\left\{u_{4}\right\}\right)\right\}$ is a soft expert $g$-closed set in $U$.

Now, one can see that every soft expert closed is soft expert $g$-closed. But the converse is not true in general. The following example supports our claim.

Example 3.3. In Example 3.2, $(J, A)$ is soft expert $g$-closed but not soft 
expert closed, where $(J, A)=\left\{\left(\left(e_{1}, p, 1\right),\left\{u_{3}\right\}\right),\left(\left(e_{1}, q, 1\right),\left\{u_{2}, u_{3}\right\}\right),\left(\left(e_{2}, p, 1\right)\right.\right.$, $\left.\left\{u_{1}, u_{2}, u_{3}\right\}\right), \quad\left(\left(e_{2}, q, 1\right),\left\{u_{2}, u_{4}\right\}\right), \quad\left(\left(e_{2}, r, 1\right),\left\{u_{3}\right\}\right), \quad\left(\left(e_{3}, p, 1\right),\left\{u_{1}, u_{2}\right\}\right)$, $\left(\left(e_{3}, q, 1\right),\left\{u_{3}, u_{4}\right\}\right),\left(\left(e_{3}, r, 1\right),\left\{u_{1}, u_{2}, u_{3}\right\}\right),\left(\left(e_{1}, p, 0\right),\left\{u_{1}, u_{2}, u_{4}\right\}\right), \quad\left(\left(e_{1}, q, 0\right)\right.$, $\left.\left\{u_{1}, u_{4}\right\}\right),\left(\left(e_{1}, r, 0\right),\left\{u_{3}, u_{4}\right\}\right),\left(\left(e_{2}, p, 0\right),\left\{u_{4}\right\}\right),\left(\left(e_{2}, q, 0\right),\left\{u_{1}, u_{3}\right\}\right),\left(\left(e_{2}, r, 0\right)\right.$, $\left.\left.\left\{u_{1}, u_{2}, u_{4}\right\}\right),\left(\left(e_{3}, p, 0\right),\left\{u_{3}, u_{4}\right\}\right),\left(\left(e_{3}, q, 0\right),\left\{u_{1}, u_{2}\right\}\right),\left(\left(e_{3}, r, 0\right),\left\{u_{4}\right\}\right)\right\}$.

Now, we establish the condition for the superset of a soft expert $g$-closed set to be a soft expert $g$-closed set.

Theorem 3.4. If $(F, A)$ is soft expert $g$-closed in $U$ and

$$
(F, A) \widetilde{\widetilde{\subseteq}}(G, A) \widetilde{\widetilde{\subseteq}} \overline{(F, A)},
$$

then $(G, A)$ is soft expert $g$-closed.

Proof. Suppose that $(F, A)$ is soft expert $g$-closed in $U$ and

$$
(F, A) \widetilde{\subseteq}(G, A) \widetilde{\widetilde{\subseteq}} \overline{(F, A)} .
$$

Let $(G, A) \widetilde{\widetilde{\subseteq}}(H, A)$ and $(H, A)$ is soft expert open in $U$. Since $(F, A) \widetilde{\subseteq}(G, A)$ and $(G, A) \underline{\widetilde{\subseteq}}(H, A)$, we have $(F, A) \underline{\widetilde{\subseteq}}(H, A)$. Hence $\overline{(F, A)} \widetilde{\subseteq}(H, A)\{$ Since $(F, A)$ is soft expert $g$-closed $\}$. Since $(G, A) \widetilde{\subseteq} \overline{(F, A)}$, we have $\overline{(G, A)} \widetilde{\subseteq} \overline{(F, A)} \widetilde{\subseteq}(H, A)$. Therefore, $(G, A)$ is soft expert $g$-closed.

Next it is observed that soft expert $g$-closed sets are closed under finite union. This is shown in the following.

Theorem 3.5. If $(F, A)$ and $(G, A)$ are soft expert $g$-closed sets then so is $(F, A) \widetilde{\cup}(G, A)$.

Proof. Suppose that $(F, A)$ and $(G, A)$ are soft expert $g$-closed sets. Let $(H, A)$ be soft expert open in $U$ and $(F, A) \widetilde{\cup}(G, A) \widetilde{\subseteq}(H, A)$. Since

$$
(F, A) \widetilde{\cup}(G, A) \widetilde{\subseteq}(H, A),
$$

we have $(F, A) \widetilde{\subseteq}(H, A)$ and $(G, A) \widetilde{\subseteq}(H, A)$. Since $(F, A)$ is soft expert open in $U$ and $(F, A)$ and $(G, A)$ are soft expert $g$-closed sets, we have $\overline{(F, A)} \widetilde{\subseteq}(H, A)$ and $\overline{(F, A)} \widetilde{\subseteq}(H, A)$. Therefore, $\overline{(F, A) \widetilde{\cup}(G, A)} \widetilde{\subseteq}\{\overline{(F, A)}\} \widetilde{\cup}\{\overline{(G, A)}\} \widetilde{\subseteq}(H, A)$. This completes the proof.

Theorem 3.6. If a set $(F, A)$ is soft expert $g$-closed in $U$, then $\overline{(F, A)} \backslash$ $(F, A)$ contains only null soft expert closed set. 
Proof. Suppose that $(F, A)$ is soft expert $g$-closed in $U$. Let $(H, A)$ be soft expert closed and $(H, A) \widetilde{\widetilde{(F, A)}} \backslash(F, A)$. Since $(H, A)$ is soft expert closed, we have $(H, A)^{\prime}$ is soft expert open. Since $(H, A) \widetilde{\widetilde{\subseteq}} \overline{(F, A)} \backslash(F, A)$, we have $(H, A) \underline{\widetilde{\subseteq}} \overline{(F, A)}$ and $(H, A) \underline{\simeq}(F, A)^{\prime}$. Hence $(F, A) \widetilde{\widetilde{C}}(H, A)^{\prime}$. Consequently $\overline{(F, A)} \subseteq(H, A)^{\prime}$ \{Since $(F, A)$ is soft expert $g$-closed in $\left.U\right\}$. Therefore, $(H, A) \widetilde{\subseteq}\{\overline{(F, A)}\}^{\prime}$. Hence $(H, A) \widetilde{\subseteq}\{\overline{(F, A)}\}^{\prime} \widetilde{\cap(F, A)}$. Hence $\overline{(F, A)} \backslash(F, A)$ contains only null soft expert closed set.

A necessary and sufficient condition for a soft expert $g$-closed set to be a soft expert closed set is established now.

Corollary 3.7. Let $(F, A)$ be soft expert $g$-closed. Then $(F, A)$ is soft expert closed if and only if $\overline{(F, A)} \backslash(F, A)$ is soft expert closed.

Proof. Suppose that $(F, A)$ is soft expert $g$-closed and soft expert closed. Since $(F, A)$ is soft expert closed, we have $\overline{(F, A)}=(F, A)$. Therefore, $\overline{(F, A)} \backslash$ $(F, A)=\widetilde{\phi}$ which is soft expert closed.

Conversely, suppose that $(F, A)$ is soft expert $g$-closed and $\overline{(F, A)} \backslash(F, A)$ is soft expert closed. Since $(F, A)$ is soft expert $g$-closed, we have $\overline{(F, A)} \backslash(F, A)$ contains only null soft expert closed set $\{$ by Theorem 3.6\}. Since $\overline{(F, A) \backslash(F, A)}$ is itself soft expert closed, we have $\overline{(F, A)} \backslash(F, A)=\widetilde{\phi}$. Therefore, $\overline{(F, A)}=$ $(F, A)$ implies that $(F, A)$ is soft expert closed.

Theorem 3.8. If $(F, A)$ is soft expert $g$-closed and $(F, A) \widetilde{\subseteq}(G, A) \widetilde{\subseteq} \overline{(F, A)}$ then $\overline{(G, A)} \backslash(G, A)$ contains only null expert closed set.

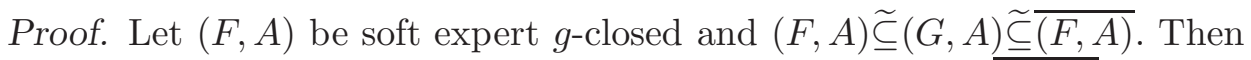
$(G, A)$ is soft expert $g$-closed \{by Theorem 3.4\}. Therefore, $\overline{(G, A) \backslash}(G, A)$ contains only null soft expert closed set.\{by Theorem 3.6

\section{Soft Expert Generalized Open Sets}

Definition 4.1. A soft expert set $(F, A)$ is called soft expert generalized open (soft expert $g$-open) if and only if $(F, A)^{\prime}$ is soft expert $g$-closed.

Example 4.2. In Example 3.2, $(F, A)$ and $(G, A)$ are soft expert $g$-open in $U$.

In the next theorem we establish a necessary and sufficient condition for a soft expert set $(F, A)$ in a soft expert topological space $U$ to be a soft expert $g$-open set. 
Theorem 4.3. A set $(F, A)$ is soft expert $g$-open if and only if $(H, A) \widetilde{\subseteq}(F, A)^{0}$ whenever $(H, A)$ is soft expert closed and $(H, A) \widetilde{\subseteq}(F, A)$.

Proof. Suppose that $(F, A)$ is soft expert $g$-open. Then, $(F, A)^{\prime}$ is soft expert $g$-closed. Suppose that $(H, A)$ is soft expert closed and $(H, A) \widetilde{\subseteq}(F, A)$. Then, $(H, A)^{\prime}$ is soft expert open and $(F, A)^{\prime} \widetilde{\subseteq}(H, A)^{\prime}$. Therefore, $\overline{(F, A)} \widetilde{\subseteq}(H, A)^{\prime}$ \{Since $(F, A)^{\prime}$ is soft expert $g$-closed\}. Since $\overline{(F, A)}=\left[(F, A)^{0}\right]^{\prime}$, we have $\left[(F, A)^{0}\right]^{\prime} \widetilde{\widetilde{\subseteq}}(H, A)^{\prime}$. Hence $(H, A) \widetilde{\subseteq}(F, A)^{0}$.

Conversely, suppose that $(H, A) \widetilde{\subseteq}(F, A)^{0}$ whenever $(H, A)$ is soft expert closed and $(H, A) \widetilde{\subseteq}(F, A)$. Then, $(F, A)^{\prime} \widetilde{\subseteq}(H, A)^{\prime}$ and $(H, A)^{\prime}$ is soft expert open. Take $(M, A)=(H, A)^{\prime}$. Since $(H, A) \widetilde{\widetilde{\subseteq}}(F, A)^{0},\left[(F, A)^{0}\right]^{\prime} \widetilde{\subseteq}(H, A)^{\prime}=(M, A)$. Since $\overline{(F, A)}=\left[(F, A)^{0}\right]^{\prime}, \overline{(F, A)} \tilde{\subseteq}(M, A)$. Therefore, $(F, A)^{\prime}$ is soft expert $g$ closed. This completes the proof.

Remark 4.4. Every soft expert open set is soft expert $g$-open but the converse is not true in general as can be seen from the following example.

Example 4.5. In Example 3.2, $(K, A)$ is soft expert $g$-open but not soft expert open where $(K, A)=\left\{\left(\left(e_{1}, p, 1\right),\left\{u_{1}, u_{2}, u_{4}\right\}\right),\left(\left(e_{1}, q, 1\right),\left\{u_{1}, u_{4}\right\}\right)\right.$, $\left(\left(e_{2}, p, 1\right),\left\{u_{4}\right\}\right),\left(\left(e_{2}, q, 1\right),\left\{u_{1}, u_{3}\right\}\right),\left(\left(e_{2}, r, 1\right),\left\{u_{1}, u_{2}, u_{4}\right\}\right),\left(\left(e_{3}, p, 1\right),\left\{u_{3}, u_{4}\right\}\right)$, $\left(\left(e_{3}, q, 1\right),\left\{u_{1}, u_{2}\right\}\right), \quad\left(\left(e_{3}, r, 1\right),\left\{u_{4}\right\}\right), \quad\left(\left(e_{1}, p, 0\right),\left\{u_{3}\right\}\right), \quad\left(\left(e_{1}, q, 0\right),\left\{u_{2}, u_{3}\right\}\right)$, $\left(\left(e_{1}, r, 0\right),\left\{u_{1}, u_{2}\right\}\right),\left(\left(e_{2}, p, 0\right),\left\{u_{1}, u_{2}, u_{3}\right\}\right),\left(\left(e_{2}, q, 0\right),\left\{u_{2}, u_{4}\right\}\right),\left(\left(e_{2}, r, 0\right),\left\{u_{3}\right\}\right)$, $\left.\left(\left(e_{3}, p, 0\right),\left\{u_{1}, u_{2}\right\}\right),\left(\left(e_{3}, q, 0\right),\left\{u_{3}, u_{4}\right\}\right),\left(\left(e_{3}, r, 0\right),\left\{u_{1}, u_{2}, u_{3}\right\}\right)\right\}$.

Next, we look at the intersection of soft expert $g$-open sets and we found that soft expert $g$-open sets are closed under finite intersection. It is shown in the following theorem.

Theorem 4.6. If $(F, A)$ and $(G, A)$ are soft expert $g$-open sets then so is $(F, A) \widetilde{\cap}(G, A)$.

Proof. Suppose that $(F, A)$ and $(G, A)$ are soft expert $g$-open sets. Let $(H, A)$ be soft expert closed and $(H, A) \widetilde{\widetilde{\subseteq}}(F, A) \widetilde{\cap}(G, A)$. Since

$$
(H, A) \widetilde{\subseteq}(F, A) \widetilde{\cap}(G, A),
$$

we have $(H, A) \widetilde{\subseteq}(F, A)$ and $(H, A) \widetilde{\subseteq}(G, A)$. Then

$$
(H, A) \widetilde{\widetilde{c}}(F, A)^{0}
$$

and

$$
(H, A) \widetilde{\subseteq}(G, A)^{0}\{\operatorname{Since}(F, A)
$$


and $(G, A)$ are soft expert $g$-open\}. Therefore,

$$
(H, A) \widetilde{\subseteq}(F, A)^{0} \widetilde{\cap}(G, A)^{0} \widetilde{\subseteq}[(F, A) \widetilde{\cap}(G, A)]^{0} .
$$

Hence $(F, A) \widetilde{\cap}(G, A)$ is soft expert $g$-open.

We now discuss about the subset of a soft expert $g$-open in $U$ in the following theorem.

Theorem 4.7. If $(F, A)$ is soft expert $g$-open in $U$ and

$$
(F, A)^{0} \widetilde{\widetilde{\subseteq}}(G, A) \widetilde{\simeq}(F, A),
$$

then $(G, A)$ is soft expert $g$-open.

Proof. Suppose that $(F, A)$ is soft expert $g$-open in $U$ and

$$
(F, A)^{0} \widetilde{\simeq}(G, A) \widetilde{\subseteq}(F, A) .
$$

Let $(H, A)$ be soft expert closed and $(H, A) \widetilde{\subseteq}(G, A)$. Since $(H, A) \widetilde{\subseteq}(G, A)$, $(G, A) \widetilde{\subseteq}(F, A)$, we have $(H, A) \widetilde{\subseteq}(F, A)$. Since $(F, A)$ is soft expert $g$-open, we have $(H, A) \widetilde{\subseteq}(F, A)^{0}$. Since $(F, A)^{0} \widetilde{\subseteq}(G, A)$, we have $(H, A) \widetilde{\subseteq}(F, A)^{0} \widetilde{\subseteq}(G, A)^{0}$. Hence $(G, A)$ is soft expert $g$-open in $U$.

Next we characterize soft expert $g$-open sets through soft expert $g$-closed sets in the following theorem.

Theorem 4.8. If a set $(F, A)$ is soft expert $g$-closed in $U, \overline{(F, A)} \backslash(F, A)$ is soft expert $g$-open.

Proof. Suppose that $(F, A)$ is soft expert $g$-closed in $U$. Let $(H, A)$ be soft expert closed and $(H, A) \widetilde{\subseteq} \overline{(F, A)} \backslash(F, A)$. Since $(F, A)$ is soft expert $g$ closed in $U$, we have $\overline{(F, A)} \backslash(F, A)$ contains only null soft expert closed set. Since $(H, A) \widetilde{\subseteq} \overline{(F, A)} \backslash(F, A)$, we have $(H, A)=\widetilde{\phi} \widetilde{\widetilde{\subseteq}}[\overline{(F, A)} \backslash(F, A)]^{0}$. Therefore, $\overline{(F, A)} \backslash(F, A)$ is soft expert $g$-open.

Theorem 4.9. If a soft expert set $(F, A)$ is soft expert $g$-open in a soft expert topological space $(U, \tau, A)$, then $(G, A)=\widetilde{U}$ whenever $(G, A)$ is soft expert open and $(F, A)^{0} \cup(F, A)^{\prime} \widetilde{\subseteq}(G, A)$.

Proof. Suppose that $(F, A)$ is soft expert $g$-open in a soft expert topological space $(U, \tau, A)$ and $(G, A)$ is soft expert open and $(F, A)^{0} \cup(F, A)^{\prime} \widetilde{\subseteq}(G, A)$. Then, $\left.(G, A)^{\prime} \widetilde{\subseteq}\left\{(F, A)^{0} \cup(F, A)^{\prime}\right\}^{\prime}=\overline{[}(F, A)^{\prime}\right] \backslash(F, A)^{\prime}$. Since $(G, A)$ is soft expert open, we have $(G, A)^{\prime}$ is soft expert closed. Since $(F, A)$ is soft expert 
$g$-open, we have $(F, A)^{\prime}$ is soft expert $g$-closed. Therefore, $\left.\overline{[}(F, A)^{\prime}\right] \backslash(F, A)^{\prime}$ contains only null soft expert closed set in $X$ by Theorem 3.6 . Consequently $(G, A)^{\prime}=\widetilde{\phi}$. Hence $(G, A)=\widetilde{U}$.

Lemma 4.10. The intersection of a soft expert $g$-open set and soft expert open set is always soft expert $g$-open.

Proof. Suppose that $(F, A)$ is soft expert $g$-open and $(G, A)$ is soft expert open. Since $(G, A)$ is soft expert open, we have $(G, A)^{\prime}$ is soft expert closed. Then $(G, A)^{\prime}$ is soft expert $g$-closed. Hence, $(G, A)$ is soft expert $g$-open. Hence $(F, A) \widetilde{\cap}(G, A)$ is soft expert $g$-open $\{$ by Theorem 4.6$\}$.

\section{References}

[1] K. Atanassov, Intuitionistic fuzzy sets, Fuzzy Sets and Systems, 20 (1986), $87-96$.

[2] K. Atanassov, Operators over interval valued intuitionistic fuzzy sets, Fuzzy Sets and Systems, 64 (1994), 159-174.

[3] D. Chen, The parametrization reduction of soft sets and its applications, Computers and Math. with Appl., 49, (2005), 757-763.

[4] W. Dunham, $T_{\frac{1}{2}}$-spaces, Kyungpook Mathematical Journal, 17 (2), (1977) 161-169.

[5] M.B. Gorzalzany, A method of inference in approximate reasoning based on interval-valued fuzzy sets, Fuzzy Sets and Systems, 21, (1987), 1-17.

[6] S. Hussain, On soft expert topological spaces, SpringerPlus (2012), 1:42. doi:10.1186/2193-1801-1-42.

[7] K. Kannan, Soft generalized closed sets in soft topological spaces, Journal of Theoretical and Applied Information Technology, 37(1), (2012), 17-21.

[8] N. Levine, Generalized closed sets topology, Rendiconti del Cirwlo Matematico di Palermo, Serie II, 19 (1970), 89-96.

[9] N. Levine, Semi-open sets and semi-continuity in topological spaces, Amer. Math. Monthly, 70 (1963), 36-41.

[10] P.K. Maji, R. Biswas, R. Roy, An application of soft sets in a decision making problem, Comput. Math. Appl., 44, (2002), 1077-1083. 
[11] P. K. Maji, R. Biswas, R. Roy, Soft set theory, Comput. Math. Appl., 45, (2003), 555-562.

[12] J. Mahanta, P. K. Das, On soft topological space via semiopen and semiclosed soft sets, arXiv math. GN 1203.4133v1.

[13] D. Molodtsov, Soft set theory first results, Comput. Math. Appl., 37, (1999), 19-31.

[14] Muhammad Shabir, Munazza Naz, On soft topological spaces, Comput. Math. Appl., 61, (2011), 1786-1799.

[15] Z. Pawlak, Rough sets, Int. J. Comput. Sci., 11, (1982), 341-356.

[16] D. Pie, D. Miao, From soft sets to information systems, Granular computing, 2005 IEEE Inter. Conf., 2, 617-621.

[17] S. Alkhazaleh and A.R. Salleh, Soft Expert Sets, Advances in Decision Sciences, Volume 2011, Article ID 757868, 12 pages doi:10.1155/2011/757868.

[18] L.A. Zadeh, Fuzzy sets, Inf. Control, 8, (1965), 338-353. 